\title{
Sufficient Conditions for the Global Rigidity of Periodic Graphs
}

\author{
Viktória E. Kaszanitzky ${ }^{1,2} \cdot$ Csaba Király $^{2,3}$ (1) $\cdot$ Bernd Schulze $^{4}$ (])
}

Received: 21 April 2018 / Revised: 12 July 2021 / Accepted: 13 July 2021 / Published online: 24 November 2021

(C) The Author(s) 2021

\begin{abstract}
Tanigawa (2016) showed that vertex-redundant rigidity of a graph implies its global rigidity in arbitrary dimension. We extend this result to periodic frameworks under fixed lattice representations. That is, we show that if a generic periodic framework is vertex-redundantly rigid, in the sense that the deletion of a single vertex orbit under the periodicity results in a periodically rigid framework, then it is also periodically globally rigid. Our proof is similar to the one of Tanigawa, but there are some added difficulties. First, it is not known whether periodic global rigidity is a generic property in dimension $d>2$. We work around this issue by using slight modifications of recent results of Kaszanitzky et al. (2021). Secondly, while the rigidity of finite frameworks in $\mathbb{R}^{d}$ on at most $d$ vertices obviously implies their global rigidity, it is non-trivial to prove a similar result for periodic frameworks. This is accomplished by extending a result of Bezdek and Connelly (2002) on the existence of a continuous motion between two equivalent $d$-dimensional realisations of a single graph in $\mathbb{R}^{2 d}$ to periodic frameworks. As an application of our result, we give a necessary and sufficient condition for the global rigidity of generic periodic body-bar frameworks in arbitrary dimension. This provides a periodic counterpart to a result of Connelly et al. (2013) regarding the global rigidity of generic finite body-bar frameworks.
\end{abstract}

Keywords Rigidity · Global rigidity · Body-bar framework · Periodic framework Mathematics Subject Classification 52C25 -05B35 - 05C10 - 68R10

\section{Introduction}

A $d$-dimensional bar-joint framework is a pair $(G, p)$, where $G$ is a simple graph and $p$ is a map which assigns a point in $\mathbb{R}^{d}$ to each vertex of $G$. We think of $(G, p)$ as a straight line realisation of $G$ in $\mathbb{R}^{d}$, where the edge lengths are measured by

Editor in Charge: János Pach

Extended author information available on the last page of the article 
the standard Euclidean metric. Loosely speaking, $(G, p)$ is (locally) rigid if any edgelength preserving continuous motion of the vertices of $(G, p)$ is necessarily a congruent motion (i.e., a motion corresponding to an isometry of $\left.\mathbb{R}^{d}\right)$. Moreover, $(G, p)$ is globally rigid if it is the only framework in $d$-space with the same graph and edge lengths, up to congruent motions. It is well known that both rigidity and global rigidity are generic properties, in the sense that a generic realisation of a graph $G$ in $\mathbb{R}^{d}$ is rigid (globally rigid) if and only if every generic realisation of $G$ in $\mathbb{R}^{d}$ is rigid (globally rigid) $[1,7,8]$. Therefore, a graph $G$ is called rigid (globally rigid) if some (equivalently any) generic realisation of $G$ is rigid (globally rigid).

The celebrated Laman's theorem from 1970 (which had previously been discovered by Pollaczek-Geiringer in 1927 [17]), gives a combinatorial characterisation of the rigid graphs in $\mathbb{R}^{2}$ [15]. Extending this result to higher dimensions is a fundamental open problem in distance geometry [27]. Similarly, a combinatorial characterisation of the globally rigid graphs in $\mathbb{R}^{2}$ has been obtained by Jackson and Jordán in 2005 [9], but the problem of extending this result to higher dimensions also remains open. For the special class of body-bar frameworks [27], however, complete combinatorial characterisations for rigidity and global rigidity have been established in all dimensions in [26] and [6], respectively.

Tanigawa recently proved the following result, which is an important new tool to investigate the global rigidity of frameworks in $\mathbb{R}^{d}$.

Theorem 1.1 ([24]) Let $G$ be a rigid graph in $\mathbb{R}^{d}$ and suppose $G-v$ remains rigid for every vertex $v$ of $G$. Then $G$ is globally rigid in $\mathbb{R}^{d}$.

In particular, the following combinatorial characterisation of globally rigid body-bar frameworks in $\mathbb{R}^{d}$ by Connelly et al. [6] easily follows from this result.

Theorem 1.2 ([6,24]) A generic body-bar framework is globally rigid in $\mathbb{R}^{d}$ if and only if it is rigid in $\mathbb{R}^{d}$ and it remains rigid after the removal of any edge.

In Sects. 4 and 5, we obtain analogues of these results for infinite periodic frameworks under fixed lattice representations. Due to their applications in fields such as crystallography, materials science, and engineering, the rigidity and flexibility of periodic structures has seen an increased interest in recent years (see e.g. $[3,4,13,16,19,21])$. In particular, combinatorial characterisations of generic rigid and globally rigid periodic bar-joint frameworks under fixed lattice representations in $\mathbb{R}^{2}$ were obtained in [21] and [13], respectively. Analogous to the situation for finite frameworks, extensions of these results to higher dimensions remain key open problems in the field. In fact, while it is well known that periodic local rigidity is a generic property in each dimension, it is currently not even known whether periodic global rigidity is a generic property for any $d>2$.

For the special class of periodic body-bar frameworks, Ross gave a combinatorial characterisation for generic local rigidity in $\mathbb{R}^{3}$ [20], and this result was recently extended to all dimensions by Tanigawa in [25] (see also Theorem 5.3). As an application of the main result of Sect. 4 (Theorem 4.5), we give the first combinatorial characterisation of generic globally rigid periodic body-bar frameworks in all dimensions in Sect. 5 (Theorem 5.2). We note that the proof of Theorem 5.2 does not rely on periodic global rigidity being a generic property in $\mathbb{R}^{d}$, and it also does not require the 
notion of stress matrices [5,6]. It is a consequence of Theorem 5.2 that global rigidity of periodic body-bar frameworks is a generic property in each dimension.

\section{Preliminaries}

\section{1 Г-Labelled Graphs and Periodic Graphs}

Let $\Gamma$ be a group isomorphic to $\mathbb{Z}^{k}$ for some integer $k>0$. A $\Gamma$-labelled graph is a pair $(G, \psi)$ of a finite directed (multi-) graph $G$ and a map $\psi: E(G) \rightarrow \Gamma$.

For a given $\Gamma$-labelled graph $(G, \psi)$, one may construct a $k$-periodic graph $\tilde{G}$ by setting $V(\tilde{G})=\left\{\gamma v_{i}: v_{i} \in V(G), \gamma \in \Gamma\right\}$ and $E(\tilde{G})=\left\{\left\{\gamma v_{i}, \psi\left(v_{i} v_{j}\right) \gamma v_{j}\right\}\right.$ : $\left.\left(v_{i}, v_{j}\right) \in E(G), \gamma \in \Gamma\right\}$. This $\tilde{G}$ is called the covering of $(G, \psi)$, and $\Gamma$ is the periodicity of $\tilde{G}$, which acts naturally on $V(\tilde{G})$ and $E(\tilde{G})$. The graph $(G, \psi)$ is also called the quotient $\Gamma$-labelled graph of $\tilde{G}$.

To guarantee that the covering of $(G, \psi)$ is a simple graph, we assume that $(G, \psi)$ has no parallel edges with the same label when oriented in the same direction. Moreover, we assume that $(G, \psi)$ has no loops. This is because a loop in $(G, \psi)$ (with a non-trivial label) does not give rise to any constraint when we study the rigidity and flexibility of the covering $\tilde{G}$ under fixed lattice representations, as will become clear below.

Note that the orientation of $(G, \psi)$ is only used as a reference orientation and may be changed, provided that we also modify $\psi$ so that if an edge has a label $\gamma$ in one direction, then it has the label $\gamma^{-1}$ in the other direction. The resulting $\Gamma$-labelled graph still has the same covering $\tilde{G}$.

It is also often useful to modify $(G, \psi)$ by using the switching operation. A switching at $v \in V(G)$ by $\gamma \in \Gamma$ changes $\psi$ to $\psi^{\prime}$ defined by $\psi^{\prime}(e)=\gamma \psi(e)$ if $e$ is directed from $v, \psi^{\prime}(e)=\gamma^{-1} \psi(e)$ if $e$ is directed to $v$, and $\psi^{\prime}(e)=\psi(e)$ otherwise. It is easy to see that a switching operation performed on a vertex in $(G, \psi)$ does not alter the covering $\tilde{G}$, up to isomorphism.

Given a $\Gamma$-labelled graph $(G, \psi)$, we define a walk in $(G, \psi)$ as an alternating sequence $v_{1}, e_{1}, v_{2}, \ldots, e_{k}, v_{k+1}$ of vertices and edges such that $v_{i}$ and $v_{i+1}$ are the endvertices of $e_{i}$. For a closed walk $C=v_{1}, e_{1}, v_{2}, \ldots, e_{k}, v_{1}$ in $(G, \psi)$, let $\psi(C)=$ $\prod_{i=1}^{k} \psi\left(e_{i}\right)^{\operatorname{sign}\left(e_{i}\right)}$, where $\operatorname{sign}\left(e_{i}\right)=1$ if $e_{i}$ has forward direction in $C$, and $\operatorname{sign}\left(e_{i}\right)=$ -1 otherwise. For a subgraph $H$ of $G$ define $\Gamma_{H}$ as the subgroup of $\Gamma$ generated by the elements $\psi(C)$, where $C$ ranges over all closed walks in $H$. The rank of $H$ is defined to be the rank of $\Gamma_{H}$. Note that the rank of $G$ may be less than the rank of $\Gamma$, in which case the covering graph $\tilde{G}$ contains an infinite number of connected components.

\subsection{Periodic Bar-Joint Frameworks}

Recall that a pair $(G, p)$ of a simple graph $G=(V, E)$ and a map $p: V \rightarrow \mathbb{R}^{d}$ is called a (bar-joint) framework in $\mathbb{R}^{d}$. A periodic framework is a special type of infinite framework defined as follows. 
Let $\tilde{G}=(\tilde{V}, \tilde{E})$ be a $k$-periodic graph with periodicity $\Gamma$, and let $L: \Gamma \rightarrow \mathbb{R}^{d}$ be a non-singular homomorphism with $k \leq d$, where $L$ is said to be non-singular if $L(\Gamma)$ has $\operatorname{rank} k$. A pair $(\tilde{G}, \tilde{p})$ of $\tilde{G}$ and $\tilde{p}: \tilde{V} \rightarrow \mathbb{R}^{d}$ is said to be an $L$-periodic framework in $\mathbb{R}^{d}$ if

$$
\tilde{p}(v)+L(\gamma)=\tilde{p}(\gamma v) \quad \text { for all } \gamma \in \Gamma \text { and all } v \in \tilde{V}
$$

We also say that a pair $(\tilde{G}, \tilde{p})$ is $k$-periodic in $\mathbb{R}^{d}$ if it is $L$-periodic for some nonsingular homomorphism $L: \Gamma \rightarrow \mathbb{R}^{d}$. Note that the rank $k$ of the periodicity may be smaller than $d$.

An $L$-periodic framework $(\tilde{G}, \tilde{p})$ is generic if the set of coordinates is algebraically independent over the rationals modulo the ideal generated by the equations (1).

A $\Gamma$-labelled framework is defined to be a triple $(G, \psi, p)$ of a finite $\Gamma$-labelled graph $(G, \psi)$ and a map $p: V(G) \rightarrow \mathbb{R}^{d}$. Given a non-singular homomorphism $L: \Gamma \rightarrow \mathbb{R}^{d}$, the covering of $(G, \psi, p)$ is the $L$-periodic framework $(\tilde{G}, \tilde{p})$, where $\tilde{G}$ is the covering of $G$ and $\tilde{p}$ is uniquely determined from $p$ by (1). $(G, \psi, p)$ is also called the quotient $\Gamma$-labelled framework of $(\tilde{G}, \tilde{p})$.

We say that a $\Gamma$-labelled framework $(G, \psi, p)$ is generic if the set of coordinates in $p$ is algebraically independent over the rationals. Note that an $L$-periodic framework $(\tilde{G}, \tilde{p})$ is generic if and only if the quotient $(G, \psi, p)$ of $(\tilde{G}, \tilde{p})$ is generic.

\subsection{Periodic Body-Bar Frameworks}

A $d$-dimensional body-bar framework consists of disjoint full-dimensional rigid bodies in $\mathbb{R}^{d}$ connected by disjoint bars, and may be considered as a special type of bar-joint framework, as we will describe below. The rigidity and flexibility of body-bar frameworks has been studied extensively (see e.g. [6,20,26,27]), as they have important applications in fields such as engineering, robotics, materials science, and biology. The underlying graph of a body-bar framework is a multi-graph $H=(V(H), E(H))$ with no loops, where each vertex in $V(H)$ corresponds to a rigid body, and each edge in $E(H)$ corresponds to a rigid bar. To represent a body-bar framework as a bar-joint framework, we extract the body-bar graph $G_{H}$ from the multi-graph $H$ as follows (see also [24], for example). $G_{H}$ is the simple graph with vertex set $V_{H}$ and edge set $E_{H}$, where

- $V_{H}$ is the disjoint union of vertex sets $B_{H}^{v}$ for each $v \in V(H)$, with $B_{H}^{v}$ defined as $B_{H}^{v}=\left\{v_{1}, v_{2}, \ldots, v_{d+1}\right\} \cup\left\{v_{e}: e \in E(H)\right.$ is incident to $\left.v\right\}$;

- $E_{H}=\left(\bigcup_{v \in V(H)} K\left(B_{H}^{v}\right)\right) \cup\left\{e^{\prime}=u_{e} v_{e}: e=u v \in E(H)\right\}$, where $K\left(B_{H}^{v}\right)$ is the complete graph on $B_{H}^{v}$.

For each $v \in V(H)$, the vertices of $B_{H}^{v}$ induce a complete subgraph of $G_{H}$, which is referred to as the body associated with $v$. A bar-joint framework $\left(G_{H}, p\right)$ with $p: V_{H} \rightarrow \mathbb{R}^{d}$ is called a body-bar realisation of $H$ in $\mathbb{R}^{d}$. See Fig. 1 for an example.

To define a periodic body-bar framework, we start with a $\Gamma$-labelled graph $(H, \psi)$, as defined in Sect. 2.1. However, we now allow $(H, \psi)$ to have loops with non-trivial labels, as well as parallel edges with equal labels when oriented in the same direction. Thus, $(H, \psi)$ defines a $k$-periodic multi-graph $\tilde{H}$ which has no loops but may have 

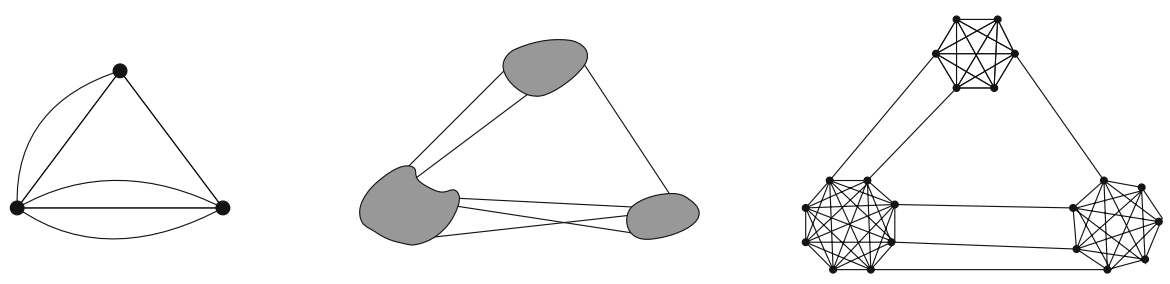

Fig. 1 Example of a 2-dimensional multi-graph $H$ (on the left) which is the underlying graph of the body-bar framework in the middle. On the right the graph $G_{H}$ is shown

parallel edges. We now use the procedure described above to construct the $k$-periodic body-bar graph $G_{\tilde{H}}$ from the multi-graph $\tilde{H}$, with the slight modification that for any edge $e \in E(\tilde{H})$ joining a vertex $v$ with $\gamma v$ for some $\gamma \neq \mathrm{id}$, we add two vertices $v_{e^{-}}$ and $v_{e^{+}}$(instead of just one vertex $v_{e}$ ) to $B_{\tilde{H}}^{v}$, and define $e^{\prime}$ to be the edge $v_{e^{-}} \gamma v_{e^{+}}$ (instead of $v_{e} \gamma v_{e}$ ). This guarantees that the quotient $\Gamma$-labelled graph of the body-bar graph $G_{\tilde{H}}$ has no loops.

An $L$-periodic bar-joint framework $\left(G_{\tilde{H}}, \tilde{p}\right)$ with $\tilde{p}: V_{\tilde{H}} \rightarrow \mathbb{R}^{d}$ is called an $L$ periodic body-bar realisation of $\tilde{H}$ in $\mathbb{R}^{d}$.

\subsection{Rigidity and Global Rigidity}

Let $G=(V, E)$ be a graph. Two bar-joint frameworks $(G, p)$ and $(G, q)$ in $\mathbb{R}^{d}$ are said to be equivalent if

$$
\|p(u)-p(v)\|=\|q(u)-q(v)\| \quad \text { for all } u v \in E .
$$

They are congruent if

$$
\|p(u)-p(v)\|=\|q(u)-q(v)\| \quad \text { for all } u, v \in V \text {. }
$$

A bar-joint framework $(G, p)$ is called globally rigid if every framework $(G, q)$ in $\mathbb{R}^{d}$ which is equivalent to $(G, p)$ is also congruent to $(G, p)$.

Analogously, following [13], we define an $L$-periodic bar-joint framework $(\tilde{G}, \tilde{p})$ in $\mathbb{R}^{d}$ to be $L$-periodically globally rigid if every $L$-periodic framework in $\mathbb{R}^{d}$ which is equivalent to $(\tilde{G}, \tilde{p})$ is also congruent to $(\tilde{G}, \tilde{p})$. Note that if the rank of the periodicity is equal to zero, then $L$-periodic global rigidity coincides with the global rigidity of finite frameworks.

A key notion to analyse $L$-periodic global rigidity is $L$-periodic rigidity. A framework $(\tilde{G}, \tilde{p})$ is called $L$-periodically rigid if there is an open neighborhood $N$ of $\tilde{p}$ in which every $L$-periodic framework $(\tilde{G}, \tilde{q})$ which is equivalent to $(\tilde{G}, \tilde{p})$ is also congruent to $(\tilde{G}, \tilde{p})$. If $(\tilde{G}, \tilde{p})$ is not $L$-periodically rigid, then it is called $L$-periodically flexible.

A bar-joint framework $(\tilde{G}, \tilde{p})$ is called L-periodically vertex-redundantly rigid, or L-periodically 2-rigid in short, if for every vertex orbit $\tilde{v}$ of $\tilde{G}$, the framework $\left(\tilde{G}-\tilde{v},\left.\tilde{p}\right|_{V(\tilde{G})-\tilde{v}}\right)$ is $L$-periodically rigid. 


\subsection{Characterisation of L-Periodic Rigidity}

A key tool to analyse the rigidity or global rigidity of finite frameworks is the lengthsquared function and its Jacobian, called the rigidity matrix. We may use the same approach to analyse periodic rigidity or periodic global rigidity (see also [13]).

For a $\Gamma$-labelled graph $(G, \psi)$ and $L: \Gamma \rightarrow \mathbb{R}^{d}$, we define $f_{G, L}: \mathbb{R}^{d|V(G)|} \rightarrow$ $\mathbb{R}^{|E(G)|}$ to be the function that assigns to every $p \in \mathbb{R}^{d|V(G)|}$ the tuple of squared edge lengths of the $\Gamma$-labelled framework $(G, \psi, p)$ (for a given order of the edges). That is, for $p \in \mathbb{R}^{d|V(G)|}$, we have

$$
f_{G, L}(p)=\left(\ldots,\left\|p\left(v_{i}\right)-\left(p\left(v_{j}\right)+L\left(\psi\left(v_{i} v_{j}\right)\right)\right)\right\|^{2}, \ldots\right) .
$$

For a finite set $V$, the complete $\Gamma$-labelled graph $K(V, \Gamma)$ on $V$ is defined to be the $\Gamma$-labelled graph with vertex set $V$ and edge set $\{((u, v) ; \gamma): u, v \in V, \gamma \in \Gamma\}$, where $((u, v) ; \gamma)$ denotes the directed edge $(u, v)$ with label $\gamma$. We simply denote $f_{K(V, \Gamma), L}$ by $f_{V, L}$. By (1) we have the following fundamental fact.

Proposition 2.1 Let $(\tilde{G}, \tilde{p})$ be an L-periodic framework and let $(G, \psi, p)$ be a quotient $\Gamma$-labelled framework of $(\tilde{G}, \tilde{p})$. Then $(\tilde{G}, \tilde{p})$ is L-periodically globally rigid (resp. rigid) if and only if for every $q \in \mathbb{R}^{d|V(G)|}$ (resp. for every $q$ in an open neighborhood of $p$ in $\left.\mathbb{R}^{d|V(G)|}\right), f_{G, L}(p)=f_{G, L}(q)$ implies $f_{V(G), L}(p)=f_{V(G), L}(q)$.

We may therefore say that a $\Gamma$-labelled framework $(G, \psi, p)$ is L-periodically globally rigid (or rigid) if for every $q \in \mathbb{R}^{d|V(G)|}$ (resp. for every $q$ in an open neighborhood of $p$ in $\left.\mathbb{R}^{d|V(G)|}\right), f_{G, L}(p)=f_{G, L}(q)$ implies $f_{V(G), L}(p)=f_{V(G), L}(q)$, and we may focus on characterising the $L$-periodic global rigidity (or rigidity) of $\Gamma$-labelled frameworks. If $(G, \psi, p)$ is not $L$-periodically rigid, then it is called $L$-periodically flexible. A $\Gamma$-labelled framework $(G, \psi, p)$ is $L$-periodically 2 -rigid if for every vertex $v$ of $G$, the $\Gamma$-labelled framework $\left(G-v,\left.\psi\right|_{G-v},\left.p\right|_{V(G)-v}\right)$ is $L$-periodically rigid. We have the following basic result for analysing $L$-periodic rigidity.

Theorem 2.2 ([13,19]) Let $(G, \psi, p)$ be a generic $\Gamma$-labelled framework in $\mathbb{R}^{d}$ with $|V(G)| \geq d+1$ and rank $k$ periodicity $\Gamma$, and let $L: \Gamma \rightarrow \mathbb{R}^{d}$ be non-singular. Then $(G, \psi, p)$ is L-periodically rigid if and only if

$$
\left.\operatorname{rank} d f_{G, L}\right|_{p}=d|V(G)|-d-\left(\begin{array}{c}
d-k \\
2
\end{array}\right),
$$

where $\left.d f_{G, L}\right|_{p}$ denotes the Jacobian of $f_{G, L}$ at $p$.

For combinatorial characterisations of generic $L$-periodically rigid or globally rigid $\Gamma$-labelled frameworks in $\mathbb{R}^{2}$, we refer the reader to [13,21] and [13], respectively. A combinatorial characterisation of generic $L$-periodically rigid body-bar frameworks in $\mathbb{R}^{d}$ has been established in [25] (see also Theorem 5.3).

\section{Rigidity Implies Global Rigidity for Small Graphs}

We first prove the following periodic counterpart of [2, Lem. 1]. 
Lemma 3.1 Let $(G, \psi)$ be the $\mathbb{Z}^{k}$-labelled graph with vertices $v_{1}, \ldots, v_{n}$ and no edges, and let $L: \mathbb{Z}^{k} \rightarrow \mathbb{R}^{d}$ be a non-singular homomorphism. Further, let $(G, \psi, p)$ and $(G, \psi, q)$ be two $\mathbb{Z}^{k}$-labelled frameworks whose coverings are the L-periodic frameworks $(\tilde{G}, \tilde{p})$ and $(\tilde{G}, \tilde{q})$ in $\mathbb{R}^{d}$.

We denote $p_{\gamma, i}=\tilde{p}\left(\gamma v_{i}\right)=p\left(v_{i}\right)+L(\gamma)$ and $q_{\gamma, i}=\tilde{q}\left(\gamma v_{i}\right)=q\left(v_{i}\right)+L(\gamma)$ for $i=1, \ldots, n$ and $\gamma \in \mathbb{Z}^{k}$. Let $\bar{p}_{\gamma, i}:[0,1] \rightarrow \mathbb{R}^{2 d}$ be the following continuous maps for $i=1, \ldots, n$ :

$$
\bar{p}_{\gamma, i}(t)=\left(\frac{p_{\gamma, i}+q_{\gamma, i}}{2}+\frac{p_{\gamma, i}-q_{\gamma, i}}{2} \cos \pi t, \frac{p_{\gamma, i}-q_{\gamma, i}}{2} \sin \pi t\right) .
$$

Then $\bar{p}_{\gamma, i}(0)=\left(p_{\gamma, i}, 0^{d}\right)$ and $\bar{p}_{\gamma, i}(1)=\left(q_{\gamma, i}, 0^{d}\right)$, where $0^{d}$ denotes the $d$ dimensional zero vector. Further, $\left|\bar{p}_{\gamma, i}(t)-\bar{p}_{\gamma^{\prime}, j}(t)\right|$ is monotone and $\bar{p}_{\gamma, i}(t)=$ $\bar{p}_{0^{k}, i}(t)+\left(L(\gamma), 0^{d}\right)$ for every $i, j \in\{1, \ldots, n\}$ and $\gamma, \gamma^{\prime} \in \mathbb{Z}^{k}$.

Proof We only prove the last equation as the other statements follow directly from [2, Lem. 1]. Observe that

$$
\begin{aligned}
\bar{p}_{\gamma, i}(t)= & \left(\frac{p_{\gamma, i}+q_{\gamma, i}}{2}+\frac{p_{\gamma, i}-q_{\gamma, i}}{2} \cos \pi t, \frac{p_{\gamma, i}-q_{\gamma, i}}{2} \sin \pi t\right) \\
= & \left(\frac{p_{0^{k}, i}+L(\gamma)+q_{0^{k}, i}+L(\gamma)}{2}+\frac{p_{0^{k}, i}+L(\gamma)-\left(q_{0^{k}, i}+L(\gamma)\right)}{2} \cos \pi t,\right. \\
& \left.\frac{p_{0^{k}, i}+L(\gamma)-\left(q_{0^{k}, i}+L(\gamma)\right)}{2} \sin \pi t\right) \\
= & \left(\frac{p_{0^{k}, i}+q_{0^{k}, i}}{2}+L(\gamma)+\frac{p_{0^{k}, i}-q_{0^{k}, i}}{2} \cos \pi t, \frac{p_{0^{k}, i}-q_{0^{k}, i}}{2} \sin \pi t\right) \\
= & \bar{p}_{0^{k}, i}(t)+\left(L(\gamma), 0^{d}\right)
\end{aligned}
$$

holds for every $i \in\{1, \ldots, n\}$ and $\gamma \in \mathbb{Z}^{k}$.

Lemma 3.1 implies the following theorem.

Theorem 3.2 Let $L: \Gamma \rightarrow \mathbb{R}^{d}$ be a non-singular homomorphism and let $(G, \psi, p)$ be a $\Gamma$-labelled framework in $\mathbb{R}^{d}$ which is not L-periodically globally rigid. Then the framework $\left(G, \psi,\left(p, 0^{d}\right)\right)$ with $\left(p, 0^{d}\right): V(G) \rightarrow \mathbb{R}^{2 d}$ is $\left(L, 0^{d}\right)$-periodically flexible in $\mathbb{R}^{2 d}$, where $\left(L, 0^{d}\right): \Gamma \rightarrow \mathbb{R}^{2 d}$ maps $\gamma \in \Gamma$ to $\left(L(\gamma), 0^{d}\right)$.

Proof Since $(G, \psi, p)$ is not $L$-periodically globally rigid, it follows from Proposition 2.1 that there exists a $\Gamma$-labelled framework $(G, \psi, q)$ whose covering $(\tilde{G}, \tilde{q})$ is equivalent but not congruent to the covering $(\tilde{G}, \tilde{p})$ of $(G, \psi, p)$. By Lemma 3.1, there exists a continuous deformation between $\left(\tilde{G},\left(\tilde{p}, 0^{d}\right)\right)$ and $\left(\tilde{G},\left(\tilde{q}, 0^{d}\right)\right)$ in $\mathbb{R}^{2 d}$ that maintains the lattice $\left(L, 0^{d}\right)$ and, by the monotonicity of the distances, also maintains the edge lengths. Therefore, this map proves that $\left(G, \psi,\left(p, 0^{d}\right)\right)$ is $\left(L, 0^{d}\right)$ periodically flexible. 
Let $(G, \psi, p)$ be a $\Gamma$-labelled framework in $\mathbb{R}^{d}, \tilde{G}$ be the covering of $(G, \psi)$, and $L: \Gamma \rightarrow \mathbb{R}^{d}$ be a non-singular homomorphism. Suppose $|V(G)| \leq d-k+1$. Observe that for $D \geq d$, the points $\left(\tilde{q}(v), 0^{D-d}\right), v \in V(\tilde{G})$, of the $\left(L, 0^{D-d}\right)$ periodic framework $\left(\tilde{\tilde{G}},\left(\tilde{q}, 0^{D-d}\right)\right)$ in $\mathbb{R}^{D}$ affinely span a space of dimension at most $|V(G)|+k-1 \leq d$. Now suppose that $(G, \psi, p)$ is $L$-periodically rigid in $\mathbb{R}^{d}$. Then it also has to be $L$-periodically globally rigid in $\mathbb{R}^{d}$. If not, then during its non-trivial continuous motion in $\mathbb{R}^{2 d}$, which is guaranteed to exist by Theorem 3.2, the points of the corresponding covering frameworks span an at most $d$-dimensional subspace, a contradiction. Hence we have the following corollary of Theorem 3.2.

Corollary 3.3 Let $(G, \psi, p)$ be a $\Gamma$-labelled framework in $\mathbb{R}^{d}$ with rank $k$ periodicity and $L: \Gamma \rightarrow \mathbb{R}^{d}$. Suppose that $(G, \psi, p)$ is L-periodically rigid and $|V(G)| \leq$ $d-k+1$. Then $(G, \psi, p)$ is also L-periodically globally rigid.

\section{2-Rigidity Implies Global Rigidity}

In this section we extend Theorem 1.1 to periodic frameworks by showing that $L$ periodic 2-rigidity, together with a rank condition on the $\Gamma$-labelled graph in the case when the framework is $d$-periodic in $\mathbb{R}^{d}$, implies $L$-periodic global rigidity. We need several lemmas. The first one is [11, Prop. 13].

Lemma 4.1 Let $f: \mathbb{R}^{d} \rightarrow \mathbb{R}^{d}$ be a polynomial map with rational coefficients and $p$ be a generic point in $\mathbb{R}^{d}$. Suppose that $\left.d f\right|_{p}$ is non-singular. Then for every $q \in$ $f^{-1}(f(p))$ we have $\overline{\mathbb{Q}(p)}=\overline{\mathbb{Q}(q)}$, where $\overline{\mathbb{Q}(p)}$ and $\overline{\mathbb{Q}(q)}$ denote the algebraic closures of $\mathbb{Q}(p)$ and $\mathbb{Q}(q)$, respectively.

Let $\Gamma$ be a group isomorphic to $\mathbb{Z}^{k}, t=\max \{d-k, 1\},(G, \psi)$ be a $\Gamma$-labelled graph with $|V(G)| \geq t$, and $L: \Gamma \rightarrow \mathbb{R}^{d}$ be non-singular. For simplicity we suppose that the linear span of $L(\Gamma)$ is $\{0\}^{d-k} \times \mathbb{R}^{k}$, the linear subspace spanned by the last $k$ coordinates. We pick any $t$ vertices $v_{1}, \ldots, v_{t}$, and define the augmented function of $f_{G, L}$ by $\hat{f}_{G, L}:=\left(f_{G, L}, g\right)$, where $g: \mathbb{R}^{d|V|} \rightarrow \mathbb{R}^{d+\left(\begin{array}{c}t \\ 2\end{array}\right)}$ is a rational polynomial map given by

$$
\begin{gathered}
g(p)=\left(p_{1}\left(v_{1}\right), \ldots, p_{d}\left(v_{1}\right), p_{1}\left(v_{2}\right), \ldots, p_{t-1}\left(v_{2}\right),\right. \\
\left.p_{1}\left(v_{3}\right), \ldots, p_{t-2}\left(v_{3}\right), \ldots, p_{1}\left(v_{t}\right)\right),
\end{gathered}
$$

where $p \in \mathbb{R}^{d|V|}$ and $p_{i}\left(v_{j}\right)$ denotes the $i$-th coordinate of $p\left(v_{j}\right)$. Augmenting $f_{G, L}$ by $g$ corresponds to "pinning down" some coordinates to eliminate trivial continuous motions. The following lemma is [13, Prop. 3.6].

Lemma 4.2 Let $(G, \psi, p)$ be a $\Gamma$-labelled framework in $\mathbb{R}^{d}$ with rank $k$ periodicity and $L: \Gamma \rightarrow \mathbb{R}^{d}$ be a non-singular homomorphism such that $L(\Gamma) \subset\{0\}^{d-k} \times \mathbb{R}^{k}$. Suppose that $p$ is generic and $|V(G)| \geq \max \{d-k, 1\}$. Then

$$
\left.\operatorname{rank} d \hat{f}_{G, L}\right|_{p}=\left.\operatorname{rank} d f_{G, L}\right|_{p}+d+\left(\begin{array}{c}
d-k \\
2
\end{array}\right) \text {. }
$$


We also need an adapted version of [13, Lem. 4.5], which is a periodic generalisation of an observation made in $[10,24]$. To state this lemma, we require the following definition.

Let $(G, \psi)$ be a $\Gamma$-labelled graph and let $v$ be a vertex of $G$. Suppose that every edge incident to $v$ is directed from $v$. For each pair of nonparallel edges $e_{1}=v u$ and $e_{2}=v w$ in $(G, \psi)$, let $e_{1} \cdot e_{2}$ be the edge from $u$ to $w$ with label $\psi(v u)^{-1} \psi(v w)$. We define $\left(G_{v}, \psi_{v}\right)$ to be the $\Gamma$-labelled graph obtained from $(G, \psi)$ by removing $v$ and inserting $e_{1} \cdot e_{2}$ (unless it is already present) for every pair of nonparallel edges $e_{1}, e_{2}$ incident to $v$.

Lemma 4.3 Let $(G, \psi, p)$ be a generic $\Gamma$-labelled framework in $\mathbb{R}^{d}$ with rank $k$ periodicity $\Gamma$ and with $|V(G)| \geq d-k+2$ and let $L: \Gamma \rightarrow \mathbb{R}^{d}$ be non-singular. Suppose that the covering $(\tilde{G}, \tilde{p})$ has a vertex $v$ with at least $d+1$ neighbours $\gamma_{0} v_{0}, \gamma_{1} v_{1}, \ldots, \gamma_{d} v_{d}$, where $v, v_{i} \in V(G)$ and $\gamma_{i} \in \Gamma$, so that the points $\tilde{p}\left(\gamma_{0} v_{0}\right), \tilde{p}\left(\gamma_{1} v_{1}\right), \ldots, \tilde{p}\left(\gamma_{d} v_{d}\right)$ affinely span $\mathbb{R}^{d}$. Suppose further that

- $\left(G-v,\left.\psi\right|_{G-v}, p^{\prime}\right)$ is L-periodically rigid in $\mathbb{R}^{d}$, with notation $p^{\prime}=\left.p\right|_{V(G)-v}$, and

- $\left(G_{v}, \psi_{v}, p^{\prime}\right)$ is L-periodically globally rigid in $\mathbb{R}^{d}$.

Then $(G, \psi, p)$ is L-periodically globally rigid in $\mathbb{R}^{d}$.

Proof We assume (by rotating the whole space if necessary) that $L(\Gamma)=\{0\}^{d-k} \times \mathbb{R}^{k}$. We pin the framework $(G, \psi, p)$ and take any $q \in \hat{f}_{G, L}^{-1}\left(\hat{f}_{G, L}(p)\right)$. Since $|V(G)| \geq$ $d-k+2>\max \{d-k, 1\}$, we may assume that $v$ is not "pinned" (i.e., $v$ is different from the vertices selected when augmenting $f_{G, L}$ to $\left.\hat{f}_{G, L}\right)$. Our goal is to show that $p=q$.

Let $p^{\prime}$ and $q^{\prime}$ be the restrictions of $p$ and $q$ to $V(G)-v$, respectively. Since $\left(G-v,\left.\psi\right|_{G-v}, p^{\prime}\right)$ is $L$-periodically rigid, we have rank $\left.d f_{G-v, L}\right|_{p^{\prime}}=d|V(G-v)|-$ $d-\left(\begin{array}{c}d-k \\ 2\end{array}\right)$ by Theorem 2.2. Then, by Lemma 4.2, we further have rank $\left.d \hat{f}_{G-v, L}\right|_{p^{\prime}}=$ $d|V(G-v)|$. Thus we can take a spanning subgraph $H$ of $G-v$ such that $\left.d \hat{f}_{H, L}\right|_{p^{\prime}}$ has linearly independent rows and is hence non-singular. Since $q^{\prime} \in \hat{f}_{H, L}^{-1}\left(\hat{f}_{H, L}\left(p^{\prime}\right)\right)$, it follows from Lemma 4.1 that $\overline{\mathbb{Q}\left(p^{\prime}\right)}=\overline{\mathbb{Q}\left(q^{\prime}\right)}$. This in turn implies that $q^{\prime}$ is generic.

Consider the edges $e_{0}=v v_{0}, e_{1}=v v_{1}, \ldots, e_{d}=v v_{d}$ in $(G, \psi)$ (all assumed to be directed from $v$ ) with respective labels $\psi\left(e_{0}\right)=\gamma_{0}, \psi\left(e_{1}\right)=\gamma_{1}, \ldots, \psi\left(e_{d}\right)=\gamma_{d}$. Note that we may have $v_{i}=v_{j}$ for some $i, j$. By switching, we may further assume that $\gamma_{0}=\mathrm{id}$. For each $1 \leq i \leq d$, let

$$
x_{i}=p\left(v_{i}\right)+L\left(\gamma_{i}\right)-p\left(v_{0}\right), \quad y_{i}=q\left(v_{i}\right)+L\left(\gamma_{i}\right)-q\left(v_{0}\right)
$$

and let $P$ and $Q$ be the $d \times d$-matrices whose $i$-th column is $x_{i}$ and $y_{i}$, respectively. Note that since $p\left(v_{i}\right)+L\left(\gamma_{i}\right)-p\left(v_{0}\right)=\tilde{p}\left(\gamma_{i} v_{i}\right)-\tilde{p}\left(v_{0}\right)$, and $q\left(v_{i}\right)+L\left(\gamma_{i}\right)-q\left(v_{0}\right)=$ $\tilde{q}\left(\gamma_{i} v_{i}\right)-\tilde{q}\left(v_{0}\right)$, and $p^{\prime}, q^{\prime}$ are generic, $x_{1}, \ldots, x_{d}$ and $y_{1}, \ldots, y_{d}$ are, respectively, linearly independent, and hence $P$ and $Q$ are both non-singular.

Let $x_{v}=p(v)-p\left(v_{0}\right)$ and $y_{v}=q(v)-q\left(v_{0}\right)$. We then have $\left\|x_{v}\right\|=\left\|y_{v}\right\|$ since $G$ has the edge $v v_{0}$ with $\psi\left(v v_{0}\right)=$ id. Due to the existence of the edge $e_{i}$ we also 
have

$$
\begin{aligned}
0= & \left\langle p\left(v_{i}\right)+L\left(\gamma_{i}\right)-p(v), p\left(v_{i}\right)+L\left(\gamma_{i}\right)-p(v)\right\rangle \\
& -\left\langle q\left(v_{i}\right)+L\left(\gamma_{i}\right)-q(v), q\left(v_{i}\right)+L\left(\gamma_{i}\right)-q(v)\right\rangle \\
= & \left\langle x_{i}-x_{v}, x_{i}-x_{v}\right\rangle-\left\langle y_{i}-y_{v}, y_{i}-y_{v}\right\rangle \\
= & \left\|x_{i}\right\|^{2}-\left\|y_{i}\right\|^{2}-2\left\langle x_{i}, x_{v}\right\rangle+2\left\langle y_{i}, y_{v}\right\rangle,
\end{aligned}
$$

where we used $\left\|x_{v}\right\|=\left\|y_{v}\right\|$. Denoting by $\delta$ the $d$-dimensional vector whose $i$-th coordinate is equal to $\left\|x_{i}\right\|^{2}-\left\|y_{i}\right\|^{2}$, the above $d$ equations can be summarized as

$$
0=\delta-2 P^{T} x_{v}+2 Q^{T} y_{v}
$$

which is equivalent to

$$
y_{v}=\left(Q^{T}\right)^{-1} P^{T} x_{v}-\frac{1}{2}\left(Q^{T}\right)^{-1} \delta .
$$

By putting this into $\left\|x_{v}\right\|^{2}=\left\|y_{v}\right\|^{2}$, we obtain

$$
\begin{aligned}
x_{v}^{T}\left(I_{d}-P Q^{-1}\left(P Q^{-1}\right)^{T}\right) x_{v} & -\left(\delta^{T} Q^{-1}\left(Q^{-1}\right)^{T} P^{T}\right) x_{v} \\
& +\frac{\delta^{T} Q^{-1}\left(Q^{-1}\right)^{T} \delta}{4}=0,
\end{aligned}
$$

where $I_{d}$ denotes the $d \times d$ identity matrix.

Note that each entry of $P$ is contained in $\mathbb{Q}\left(p^{\prime}\right)$, and each entry of $Q$ is contained in $\mathbb{Q}\left(q^{\prime}\right)$. Since $\overline{\mathbb{Q}\left(p^{\prime}\right)}=\overline{\mathbb{Q}\left(q^{\prime}\right)}$, this implies that each entry of $P Q^{-1}$ is contained in $\overline{\mathbb{Q}\left(p^{\prime}\right)}$. On the other hand, since $p$ is generic, the set of coordinates of $p(v)$ (and hence those of $\left.x_{v}\right)$ is algebraically independent over $\overline{\mathbb{Q}\left(p^{\prime}\right)}$. Therefore, by regarding the left-hand side of (3) as a polynomial in $x_{v}$, the polynomial must be identically zero. In particular, we get

$$
I_{d}-P Q^{-1}\left(P Q^{-1}\right)^{T}=0 \text {. }
$$

Thus, $P Q^{-1}$ is orthogonal. In other words, there is some orthogonal matrix $S$ such that $P=S Q$, and we get

$$
\left\|p\left(v_{i}\right)+L\left(\gamma_{i}\right)-p\left(v_{0}\right)\right\|=\left\|x_{i}\right\|=\left\|S y_{i}\right\|=\left\|y_{i}\right\|=\left\|q\left(v_{i}\right)+L\left(\gamma_{i}\right)-q\left(v_{0}\right)\right\|
$$

for every $1 \leq i \leq d$. Therefore, $q^{\prime} \in f_{G_{v}, L}^{-1}\left(f_{G_{v}, L}\left(p^{\prime}\right)\right)$. Since $\left(G_{v}, \psi_{v}, p\right)$ is $L$ periodically globally rigid, this in turn implies that $f_{V-v, L}\left(p^{\prime}\right)=f_{V-v, L}\left(q^{\prime}\right)$. Thus we have $p^{\prime}=q^{\prime}$.

Since $\left\{p\left(v_{i}\right)+L\left(\gamma_{i}\right): 0 \leq i \leq d\right\}$ affinely spans $\mathbb{R}^{d}$, there is a unique extension of $p^{\prime}: V(G)-v \rightarrow \mathbb{R}^{d}$ to $r: V(G) \rightarrow \mathbb{R}^{d}$ such that $f_{G, L}(r)=f_{G, L}(p)$. Thus we obtain $p=q$. 

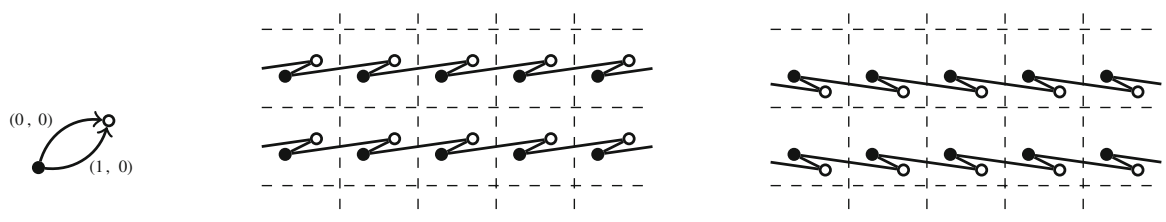

Fig. 2 Example of a $\mathbb{Z}^{2}$-labelled graph $(G, \psi)$ with $\operatorname{rank}(G, \psi)=1$ (on the left) and two equivalent but not congruent $L$-periodic frameworks $(\tilde{G}, \tilde{p})$ and $(\tilde{G}, \tilde{q})$ with rank 2 periodicity in $\mathbb{R}^{2}$

Finally, we need the following special case of [13, Lem. 3.1].

Lemma 4.4 Let $(G, \psi, p)$ be a generic $\Gamma$-labelled framework in $\mathbb{R}^{d}$ with $|V(G)| \geq 2$, rank $k$ periodicity $\Gamma$, and let $L: \Gamma \rightarrow \mathbb{R}^{d}$ be a non-singular homomorphism. If $(G, \psi, p)$ is L-periodically globally rigid, then the rank of $(G, \psi)$ is equal to $k$.

Lemma 4.4 is easily seen to be true, because if the rank of $(G, \psi)$ is less than $k$, then the covering $(\tilde{G}, \tilde{p})$ of $(G, \psi, p)$ has infinitely many connected components, each of which may be 'flipped' individually in a periodic fashion to obtain an $L$-periodic framework $(\tilde{G}, \tilde{q})$ which is equivalent, but not congruent to $(\tilde{G}, \tilde{p})$. Thus, $(G, \psi, p)$ is not $L$-periodically globally rigid.

This is illustrated by the two equivalent but non-congruent 2-periodic frameworks in $\mathbb{R}^{2}$ shown in Fig. 2 whose $\Gamma$-labelled graph $(G, \psi)$ has rank 1 . Note, however, that $(G, \psi)$ is $L$-periodically 2 -rigid, since it is $L$-periodically rigid and the removal of any vertex results in a trivial framework with one vertex orbit and no edges (recall also Theorem 2.2).

It follows that in the case when $k=d, L$-periodic 2-rigidity is not sufficient for $L$ periodic global rigidity. In this case we need the added assumption that $\operatorname{rank}(G, \psi)=$ $d$. In the case when $k<d$ and $\operatorname{rank}(G, \psi)<k,(G, \psi, p)$ can also not be $L$ periodically globally rigid, by Lemma 4.4. However, in this case, $(G, \psi, p)$ is also not $L$-periodically 2-rigid.

Theorem 4.5 Let $(G, \psi, p)$ be a generic $\Gamma$-labelled framework in $\mathbb{R}^{d}$ with rank $k$ periodicity $\Gamma$, and let $L: \Gamma \rightarrow \mathbb{R}^{d}$ be non-singular. If $(G, \psi, p)$ is L-periodically 2-rigid, and if $(G, \psi)$ is also of rank $d$ in the case when $k=d$, then $(G, \psi, p)$ is L-periodically globally rigid in $\mathbb{R}^{d}$.

Proof We use induction on $|V(G)|$. If $|V(G)| \leq d-k+1$, then $(G, \psi, p)$ is $L$ periodically globally rigid by the $L$-periodic rigidity of $(G, \psi, p)$ and Corollary 3.3.

Now suppose that $|V(G)| \geq d-k+2$, and let $(\tilde{G}, \tilde{p})$ be the covering of $(G, \psi, p)$. By our assumption, $\left(G-v,\left.\psi\right|_{G-v},\left.p\right|_{V(G)-v}\right)$ is $L$-periodically rigid for any vertex $v \in V(G)$.

Suppose first that $|V(G)|=d-k+2$. Then $\left(G-v,\left.\psi\right|_{G-v},\left.p\right|_{V(G)-v}\right)$ is also $L$-periodically globally rigid by Corollary 3.3. We claim that for any occurrence of any $v \in V(G)$ in the covering $\tilde{G}$, the affine span of the set $\{\tilde{p}(w): v w \in E(\tilde{G})\}$ is all of $\mathbb{R}^{d}$.

If $d=k$ (and hence $|V(G)|=d-k+2=2$ ) the claim follows from the fact that $\operatorname{rank}(G, \psi)=d$, by our assumption. 
If $d>k$ (and hence $|V(G)|=d-k+2>2$ ), then we suppose for a contradiction that the claim is not true. Then the removal of a neighbour of $v$ (and of all vertices belonging to that same vertex orbit) results in an $L$-periodic framework with at least two distinct orbits of points (since $|V(G)|>2$ ) and, by our genericity assumption, this framework has the property that all the points connected to $\tilde{p}(v)$ affinely span a space of dimension at most $d-2$, so that $\tilde{p}(v)$ can be rotated about this $(d-2)$-dimensional axis. Since all copies of points in the same orbit can then also be rotated in a periodic fashion and the affine span of the non-moving points is $(d-1)$-dimensional (as a $k$-periodic configuration with $d-k$ vertex orbits), we obtain a contradiction to the $L$-periodic 2-rigidity of $(\tilde{G}, \tilde{p})$.

Thus, the affine span of the points $\{\tilde{p}(w): v w \in E(\tilde{G})\}$ is indeed all of $\mathbb{R}^{d}$ as claimed, and it follows from Lemma 4.3 that $(G, \psi, p)$ is $L$-periodically globally rigid.

We may therefore assume that $|V(G)|>d-k+2$. We show that $\left(G_{v}, \psi_{v}, p^{\prime}\right)$ is $L$-periodically 2-rigid for any $v \in V(G)$. Suppose for a contradiction that this is not true. Then there is a vertex $u$ whose removal results in an $L$-periodically flexible framework. As the neighbours of one occurrence of $v$ in $\tilde{G}$ induce a complete graph in $\tilde{G}_{v}$ (where any pair of vertices from the same vertex orbit may always be considered adjacent due to the fixed lattice representation), adding $v$ together with its incident edges to $\left(G_{v}-u,\left.\psi_{v}\right|_{G_{v}-u},\left.p\right|_{V(G)-\{u, v\}}\right)$ still yields an $L$-periodically flexible framework. This is a contradiction, as $\left(G-u,\left.\psi\right|_{G-u},\left.p\right|_{V(G)-u}\right)$ is an $L-$ periodically rigid $\Gamma$-labelled spanning subframework of the framework obtained from $\left(G_{v}-u,\left.\psi_{v}\right|_{G_{v}-u},\left.p\right|_{V(G)-\{u, v\}}\right)$ by adding $v$ and its incident edges.

Thus $\left(G_{v}, \psi_{v}, p^{\prime}\right)$ is $L$-periodically 2 -rigid as claimed. Moreover, since $(G, \psi)$ is 2-connected by the $L$-periodic 2-rigidity of $(G, \psi, p)$, it follows from the definition of $\left(G_{v}, \psi_{v}\right)$ that $\Gamma_{G}=\Gamma_{G_{v}}$. Thus, if $(G, \psi)$ is of rank $d$ then so is $\left(G_{v}, \psi_{v}\right)$. It now follows from the induction hypothesis that $\left(G_{v}, \psi_{v}, p^{\prime}\right)$ is $L$-periodically globally rigid. Moreover, by the same argument as above for the case when $|V(G)|=d-$ $k+2>2$, the affine span of the points $\{\tilde{p}(w): v w \in E(\tilde{G})\}$ is all of $\mathbb{R}^{d}$. Thus, by Lemma $4.3,(G, \psi, p)$ is $L$-periodically globally rigid.

\section{Global Rigidity of Body-Bar Frameworks}

Using Theorem 4.5 in combination with Lemma 4.4 and the following Lemma 5.1 (which is [13, Lem. 3.7]) we can now easily prove an extension of Theorem 1.2 to periodic body-bar frameworks. We need the following definitions.

An $L$-periodic framework $(\tilde{G}, \tilde{p})$ with rank $k$ periodicity $\Gamma$ is said to be $L$ periodically bar-redundantly rigid if $(\tilde{G}-\tilde{e}, \tilde{p})$ is $L$-periodically rigid for every edge orbit $\tilde{e}$ of $\tilde{G}$.

Similarly, an $L$-periodic body-bar realisation $\left(G_{\tilde{H}}, \tilde{p}\right)$ of a multi-graph $\tilde{H}$ is $L$ periodically bar-redundantly rigid if for every edge orbit $\tilde{e}$ of $\tilde{H}$, the framework $\left(G_{\tilde{H}}-\tilde{e}, \tilde{p}\right)$ is $L$-periodically rigid. (Recall the definition of a body-bar realisation in Sect. 2.3.) 
Lemma 5.1 ([13]) Let $(\tilde{G}, \tilde{p})$ be a generic L-periodic framework in $\mathbb{R}^{d}$ with rank $k$ periodicity $\Gamma$, and let $L: \Gamma \rightarrow \mathbb{R}^{d}$ be non-singular. Suppose also that the quotient $\Gamma$-labelled graph $(G, \psi)$ of $\tilde{G}$ has $|V(G)| \geq d+1$ if $k \geq 1$ and $|V(G)| \geq d+2$ if $k=0$. If $(\tilde{G}, \tilde{p})$ is L-periodically globally rigid, then $(\tilde{G}, \tilde{p})$ is L-periodically bar-redundantly rigid.

The following extension of Theorem 1.2 gives a combinatorial characterisation of generic $L$-periodically globally rigid body-bar frameworks in $\mathbb{R}^{d}$.

Theorem 5.2 Let $\left(G_{\tilde{H}}, \tilde{p}\right)$ be a generic L-periodic body-bar realisation of the multigraph $\tilde{H}$ in $\mathbb{R}^{d}$ with rank $k$ periodicity $\Gamma$, and let $L: \Gamma \rightarrow \mathbb{R}^{d}$ be non-singular. Then $\left(G_{\tilde{H}}, \tilde{p}\right)$ is L-periodically globally rigid in $\mathbb{R}^{d}$ if and only if $\left(G_{\tilde{H}}, \tilde{p}\right)$ is L-periodically bar-redundantly rigid in $\mathbb{R}^{d}$, and the quotient $\Gamma$-labelled graph of $G_{\tilde{H}}$ is of rank $d$ in the case when $k=d$.

Proof It immediately follows from Lemma 5.1 that $L$-periodic bar-redundant rigidity is necessary for a generic $L$-periodic body-bar realisation to be $L$-periodically globally rigid. Moreover, it follows from Lemma 4.4 that in the case when $k=d$, the rank of the quotient $\Gamma$-labelled graph of $G_{\tilde{H}}$ must be equal to $d$ for a generic $L$-periodic body-bar realisation to be $L$-periodically globally rigid. It is also easy to see that if a generic $L$-periodic body-bar realisation is $L$-periodically bar-redundantly rigid, then it is $L$-periodically 2-rigid, since the edges connecting the bodies are all disjoint. The result now follows from Theorem 4.5.

Note that generic $L$-periodic bar-redundant rigidity can easily be checked in polynomial time based on the combinatorial characterisation of generic $L$-periodic rigidity of body-bar frameworks in $\mathbb{R}^{d}$ conjectured by Ross in [20, Conj. 5.1] and proved by Tanigawa in [25, Thm. 7.2]. Using our notation and a simplified expression for the dimension of the space of trivial motions for a $k$-periodic framework in $\mathbb{R}^{d}$, this result may be restated as follows.

Theorem 5.3 ([25]) Let $\left(G_{\tilde{H}}, \tilde{p}\right)$ be a generic L-periodic body-bar realisation of the multi-graph $\tilde{H}$ in $\mathbb{R}^{d}$ with rank $k$ periodicity $\Gamma$, and $L: \Gamma \rightarrow \mathbb{R}^{d}$ be non-singular. Then $\left(G_{\tilde{H}}, \tilde{p}\right)$ is L-periodically rigid in $\mathbb{R}^{d}$ if and only if the quotient $\Gamma$-labelled graph $H$ of $\tilde{H}$ contains a spanning subgraph $(V, E)$ satisfying the following counts:

$$
\begin{aligned}
& -|E|=\left(\begin{array}{c}
d+1 \\
2
\end{array}\right)|V|-d-\left(\begin{array}{c}
d-k \\
2
\end{array}\right) ; \\
& \text { - }|F| \leq\left(\begin{array}{c}
d+1 \\
2
\end{array}\right)|V(F)|-d-\left(\begin{array}{c}
d-k(F) \\
2
\end{array}\right) \text { for all non-empty } F \subseteq E,
\end{aligned}
$$

where $k(F)$ is the rank of $F$.

\section{Conclusion and Further Comments}

Real-world structures, whether they are natural such as crystals or proteins, or man-made such as buildings or linkages, are usually non-generic, and often exhibit 
non-trivial symmetries. This fact has motivated a significant amount of research in recent years on how symmetry impacts the rigidity and flexibility of frameworks (see [23], for example, for a summary of results). In Theorem 4.5, we have shown that the sufficient condition given by Tanigawa in [24] for generic global rigidity of finite frameworks can be transformed to a sufficient condition for generic global rigidity of infinite $L$-periodic frameworks (under a fixed lattice $L$ ). It remains open whether this result can be extended to other types of frameworks with symmetries such as infinite periodic frameworks with (partially) flexible lattices or finite frameworks with point group symmetries. Following the proof of Theorem 5.2, such an extension would imply the characterisation of the generic global rigidity of finite body-bar frameworks with these symmetries by using the existing (local) rigidity characterisations of these frameworks by Tanigawa [25]. Furthermore, such a result would be useful for the characterisation of the generic global rigidity of body-hinge frameworks with symmetries (where the bodies are connected in pairs by $(d-2)$-dimensional hinges) such as in the (finite) generic version established by Jordán, Király, and Tanigawa [12]. However, the characterisation of generic (local) rigidity for periodic body-hinge frameworks is still open (even for fixed lattices). For finite symmetric body-hinge frameworks, such a characterisation is only known for groups of the form $\mathbb{Z}_{2} \times \mathbb{Z}_{2} \times \cdots \times \mathbb{Z}_{2}$ [22]. A major goal in this research area is to obtain a combinatorial characterisation of the generic global rigidity of infinite $L$-periodic or finite symmetric molecular frameworks in 3-space (i.e., body-hinge frameworks in 3-space with the added property that the lines of the hinges attached to each body all meet in a single point on that body), since they may be used to model crystals and protein structures. We note that for finite molecular frameworks, their generic (local) rigidity was recently characterised by the celebrated result of Katoh and Tanigawa [14]. However, their generic global rigidity has not yet been characterised, and there are also no generic local or global rigidity characterisations for infinite $L$-periodic or finite symmetric molecular frameworks [18].

Acknowledgements Project No. NKFI-128673 has been implemented with the support provided from the National Research, Development and Innovation Fund of Hungary, financed under the FK_18 funding scheme (Kaszanitzky and Király). The first author was supported by the Hungarian Scientific Research Fund of the National Research, Development and Innovation Office (OTKA, Grant Number K109240 and K124171). The second author was supported by the János Bolyai Research Scholarship of the Hungarian Academy of Sciences, by the ÚNKP-17- 4 New National Excellence Program of the Ministry of Human Capacities of Hungary, by the ÚNKP-20-5 New National Excellence Program of the Ministry for Innovation and Technology, and by the Hungarian Scientific Research Fund of the National Research, Development and Innovation Office (OTKA, Grant Number K109240). The third author was supported by EPSRC First Grant EP/M013642/1.

Open Access This article is licensed under a Creative Commons Attribution 4.0 International License, which permits use, sharing, adaptation, distribution and reproduction in any medium or format, as long as you give appropriate credit to the original author(s) and the source, provide a link to the Creative Commons licence, and indicate if changes were made. The images or other third party material in this article are included in the article's Creative Commons licence, unless indicated otherwise in a credit line to the material. If material is not included in the article's Creative Commons licence and your intended use is not permitted by statutory regulation or exceeds the permitted use, you will need to obtain permission directly from the copyright holder. To view a copy of this licence, visit http://creativecommons.org/licenses/by/4.0/. 


\section{References}

1. Asimow, L., Roth, B.: The rigidity of graphs. Trans. Am. Math. Soc. 245, 279-289 (1978)

2. Bezdek, K., Connelly, R.: Pushing disks apart-the Kneser-Poulsen conjecture in the plane. J. Reine Angew. Math. 553, 221-236 (2002)

3. Borcea, C.S., Streinu, I.: Periodic frameworks and flexibility. Proc. R. Soc. Lond. Ser. A 466(2121), 2633-2649 (2021)

4. Borcea, C.S., Streinu, I., Tanigawa, S.: Periodic body-and-bar frameworks. SIAM J. Discrete Math. 29(1), 93-112 (2015)

5. Connelly, R.: Rigidity and energy. Invent. Math. 66(1), 11-33 (1982)

6. Connelly, R., Jordán, T., Whiteley, W.: Generic global rigidity of body-bar frameworks. J. Comb. Theory Ser. B 103(6), 689-705 (2013)

7. Gluck, H.: Almost all simply connected closed surfaces are rigid. In: Geometric Topology (Park City 1974). Lecture Notes in Math., vol. 438, pp. 225-239. Springer, Berlin (1975)

8. Gortler, S.J., Healy, A.D., Thurston, D.P.: Characterizing generic global rigidity. Am. J. Math. 132(4), 897-939 (2010)

9. Jackson, B., Jordán, T.: Connected rigidity matroids and unique realizations of graphs. J. Comb. Theory Ser. B 94(1), 1-29 (2005)

10. Jackson, B., Jordán, T., Szabadka, Z.: Globally linked pairs of vertices in equivalent realizations of graphs. Discrete Comput. Geom. 35(3), 493-512 (2006)

11. Jackson, B., Jordán, T., Tanigawa, S.: Unique low rank completability of partially filled matrices. J. Comb. Theory Ser. B 121, 432-462 (2016)

12. Jordán, T., Király, Cs., Tanigawa, S.: Generic global rigidity of body-hinge frameworks. J. Comb. Theory Ser. B 117, 59-76 (2016)

13. Kaszanitzky, V.E., Schulze, B., Tanigawa, S.: Global rigidity of periodic graphs under fixed-lattice representations. J. Comb. Theory Ser. B 146, 176-218 (2021)

14. Katoh, N., Tanigawa, S.: A proof of the molecular conjecture. Discrete Comput. Geom. 45(4), 647-700 (2011)

15. Laman, G.: On graphs and rigidity of plane skeletal structures. J. Eng. Math. 4, 331-340 (1970)

16. Malestein, J., Theran, L.: Generic combinatorial rigidity of periodic frameworks. Adv. Math. 233, 291-331 (2013)

17. Pollaczek-Geiringer, H.: Über die Gliederung ebener Fachwerke. Z. Angew. Math. Mech. 7(1), 58-72 (1927)

18. Porta, J., Ros, L., Schulze, B., Sljoka, A., Whiteley, W.: On the symmetric molecular conjectures. In: Computational Kinematics (Barcelona 2013). Mechanisms and Machine Science, vol. 15, pp. 175-184. Springer, Heidelberg-New York-London (2014)

19. Ross, E.: The rigidity of periodic frameworks as graphs on a fixed torus. Contrib. Discrete Math. 9(1), $11-45$ (2014)

20. Ross, E.: The rigidity of periodic body-bar frameworks on the three-dimensional fixed torus. Philos. Trans. R. Soc. Lond. Ser. A 372(2008), \# 20120112 (2014)

21. Ross, E.: Inductive constructions for frameworks on a two-dimensional fixed torus. Discrete Comput. Geom. 54(1), 78-109 (2015)

22. Schulze, B., Tanigawa, S.: Linking rigid bodies symmetrically. Eur. J. Comb. 42, 145-166 (2014)

23. Schulze, B., Whiteley, W.: Rigidity of symmetric frameworks. In: Handbook of Discrete and Computational Geometry, 3rd edn., pp. 1633-1660. CRC Press, Boca Raton (2017)

24. Tanigawa, S.: Sufficient conditions for the global rigidity of graphs. J. Comb. Theory Ser. B 113, 123-140 (2015)

25. Tanigawa, S.: Matroids of gain graphs in applied discrete geometry. Trans. Am. Math. Soc. 367(12), 8597-8641 (2015)

26. Tay, T.-S.: Rigidity of multigraphs. I. Linking rigid bodies in $n$-space. J. Comb. Theory Ser. B 36(1), 95-112 (1984)

27. Whiteley, W.: Some matroids from discrete applied geometry. In: Matroid Theory (Seattle 1995). Contemp. Math., vol. 197, pp. 171-311. Amer. Math. Soc., Providence (1996)

Publisher's Note Springer Nature remains neutral with regard to jurisdictional claims in published maps and institutional affiliations. 


\section{Affiliations}

Viktória E. Kaszanitzky ${ }^{1,2} \cdot$ Csaba Király $^{2,3}{ }^{(0)} \cdot$ Bernd Schulze $^{4}$

Viktória E. Kaszanitzky

kaszanitzky@cs.bme.hu

Csaba Király

cskiraly@cs.elte.hu

Bernd Schulze

b.schulze@lancaster.ac.uk

1 Budapest University of Technology and Economics, Magyar tudósok krt 2., Budapest 1117, Hungary

2 The MTA-ELTE Egerváry Research Group on Combinatorial Optimization, Eötvös Loránd Research Network (ELKH), Pázmány Péter sétány 1/C, Budapest 1117, Hungary

3 Department of Operations Research, ELTE Eötvös Loránd University, Pázmány Péter sétány 1/C, Budapest 1117, Hungary

4 Department of Mathematics and Statistics, Lancaster University, Lancaster LA1 4YF, UK 